\title{
Recursos didácticos digitales que permiten mejorar la práctica pre profesional en el contexto de la emergencia sanitaria Covid-19
}

\author{
Mónica Priscila Ávila Larriva ${ }^{1}$ \\ ${ }^{1}$ Universidad Católica de Cuenca, priscila.avila@ucacue.edu.ec, Av.Américas y Humboldt S/N, Cuenca, \\ Ecuador (+593)0999280140. \\ Información del artículo revisado por pares \\ Fecha de aceptación: junio-2021 \\ Fecha de publicación en línea: diciembre-2021 \\ DOI: https://doi.org/10.29105/vtga7.1-80
}

\begin{abstract}
Resumen
La problemática del estudio se desarrolla a partir de la emergencia sanitaria Covid-19 modificando la modalidad de estudios universitarios, ésta cambia por una modalidad virtual obligando a realizar la práctica pre profesional (PPP) desde este contexto. El objetivo del estudio fue establecer recursos didácticos digitales que favorezcan la práctica PPP. La metodología del estudio es de tipo exploratorio, descriptivo, correlacional, no experimental de corte transeccional y explicativo mediante la aplicación de una encuesta a 19 estudiantes y dos tutores de la PPP. La hipótesis declara que los recursos didácticos que permiten mejorar la PPP en el contexto de la pandemia son: el portafolio digital, el juego-trabajo, la animación a la lectura mediante E-books y los bits de inteligencia en Web-albums como estrategias para el conocimiento. Los resultados fueron estadísticamente significativos, confirman la hipótesis de estudio para la ejecución de los recursos didácticos digitales en la PPP.
\end{abstract}

Palabras clave: recursos didácticos, digitales, práctica pre profesional.

\begin{abstract}
The problem of work developed from the Covid19 global health emergency impacts on higher education modality, which is modified by a virtual proposal, forcing the pre-professional practice (PPP) to be carried out from this context. This study objective was to establish digital didactic resources to favor the PPP practice. The methodology used is exploratory, descriptive, correlational, non-experimental, trans-sectional, and explanatory. It was carried out through a survey applying to 19 students and two PPP tutors. The hypothesis states that the didactic resources that can improve the PPP as knowledge development tools in the pandemic context are: the digital portfolio, the game-work, reading motivation using E-books and the intelligence bits in Web-albums. Results were statistically significant; they confirm the study hypothesis for the implementation of digital didactic resources in PPP development.
\end{abstract}

Keywords: didactic resources, digital, preprofessional practice

JEL: I20. 


\section{INTRODUCCIÓN}

A partir del mes de marzo de 2020 con la emergencia sanitaria COVID-19 las Instituciones de Educación Superior del Ecuador (IES) se vieron obligadas a modificar la modalidad de su oferta académica, pasando de una modalidad presencial tradicional a una virtual, en línea. El Consejo de Educación Superior (CES, 2020) organismo regulador de las IES emite la "Normativa transitoria para el desarrollo de actividades académicas en las Instituciones de Educación Superior, debido al estado de excepción decretado por la emergencia sanitaria ocasionada por la pandemia de COVID-19" con la finalidad de velar por el derecho a la educación en situación de vulnerabilidad y dar así una salida a la difícil situación del país. Por lo que los planes de estudio sufren ajustes especialmente en lo referente a los recursos didácticos que serían acorde a la realidad inmediata de docentes y estudiantes.

En su estudio Labra y Fuentealba (2011) sostienen que los docentes requieren de un proceso de aprendizaje producto de su práctica, ya que la enseñanza demanda de una planificación, improvisación para la consecución de los aprendizajes, la práctica permite el desarrollo significativo del conocimiento y la reflexión de los procesos. Este estudio se enfoca en los estudiantes de quinto y séptimo ciclo de la licenciatura de Educación Inicial de la Universidad Católica de Cuenca, así como también en ajustar a la PPP los recursos didácticos digitales dentro del contexto de vulnerabilidad en el que se desarrollan los procesos educativos debido a la pandemia. Es necesario analizar el nivel de accesibilidad y de conectividad en la PPP, así como la brecha digital presente a la fecha de los acontecimientos, la ubicación de los espacios para la práctica y la adaptación de los nuevos escenarios en los que se desarrollará la misma.

En el estudio de Bizberge y Segura (2020) el acceso a la conectividad en América Latina tuvo una notable disminución en la velocidad de la banda ancha debido a las medidas preventivas de aislamiento social y la necesidad de cubrir la demanda de accesibilidad a servicios de internet para: teletrabajo, clases en línea, comercio on line, se presenta el incremento del uso de la red en un $25 \%$, en Chile y Ecuador.

Torres (2020) en su estudio presenta el siguiente análisis sobre la accesibilidad y la conectividad de los estudiantes a nivel país. Existen 4.374.799 estudiantes entre educación básica y bachillerato, 3 millones (75\%) van al sistema público, asistiendo a 150.000 planteles educativos. De ellos, 2 millones tienen conectividad; 1 millón no tiene computadora ni internet en sus casas o en sus móviles. De las 12.863 unidades educativas fiscales y fiscomisionales que hay en el país, 4.747 tienen acceso a internet (datos del Ministerio de Educación, 2020), de igual manera este estudio referencia los datos del Instituto Nacional de Estadísticas y Censos (INEC, 2018) en relación con el equipamiento en hogares ecuatorianos con computador antes de la pandemia, que era del $11,2 \%$ lo que indica la falta notable de acceso a herramientas telemáticas. Las medidas que se deben tener en cuenta para la continuidad educativa de acuerdo con el estudio de la división de educación del BID en mayo de 2020, a nivel de los países de América Latina y el Caribe son: plataformas de aprendizaje, contenido digital, material físico o redes sociales, $\mathrm{TV}$ o radio y escuelas abiertas en ese orden. Los hechos actuales que contextualizan el problema de estudio; a nivel social, económico y educativo especialmente son:

El cambio de modalidad presencial a la modalidad en línea de todo el sistema educativo, el ajuste de horas de PPP, la adaptación de los nuevos escenarios para la práctica, la capacitación a docentes, estudiantes y padres de familia para el uso de herramientas y recursos digitales, la accesibilidad y conectividad, la inminente brecha digital visible a raíz de la pandemia y la ubicación de espacios o centros de educación inicial para la PPP.

Entre las causas presentes dentro del estudio fueron el portafolio digital, el juegotrabajo como medio interactivo de aprendizaje, la animación a la lectura mediante E-books, los bits de inteligencia en Web-albums. Son recursos didácticos digitales que permiten desarrollar las nociones, destrezas y habilidades de los niños y niñas dentro del escenario de la PPP, como consecuencia, los recursos propuestos son un aporte sustancial. 
La problemática del estudio se desarrolló a partir de la emergencia sanitaria COVID-19 la que impacta en la modalidad de estudios universitarios, ésta se modifica por una propuesta virtual obligando a realizar la práctica pre profesional (PPP) desde este contexto. La pregunta de estudio fue ¿Cuáles son los recursos didácticos digitales que pueden optimizar la práctica laboral o pre profesional de los estudiantes de la licenciatura de educación inicial en el contexto de la emergencia sanitaria covid19 ? Se establece como hipótesis que los recursos didácticos que permiten mejorar la práctica laboral de los estudiantes de la licenciatura de educación inicial de la Universidad Católica de Cuenca, en el contexto de la emergencia sanitaria COVID19 son: el portafolio digital, el juego-trabajo como medio interactivo de aprendizaje, la animación a la lectura mediante E-books y los bits de inteligencia en Web-albums.

Ésta investigación beneficia a los estudiantes y docentes-tutores de la licenciatura de Educación Inicial de la Universidad Católica de Cuenca, a los niños y niñas de 3 a 5 años y de manera general al sistema educativo, se aplicó el método científico que justifica éste proyecto. Teóricamente el estudio se sustenta con las investigaciones aplicadas hacia la potencialización de la PPP en la modalidad de estudio virtual y en línea, será aplicada en la ciudad de Cuenca-Ecuador, mediante un tipo de investigación exploratoria, descriptiva, correlacional, no experimental y de corte transeccional explicativa mediante la aplicación de una encuesta a 19 estudiantes y dos tutores de la PPP, de la Licenciatura de Educación Inicial, de la Universidad Católica de Cuenca en su sedematriz de la ciudad de Cuenca-Ecuador, encuestados en el segundo semestre del 2020. Para ello, se realizó un muestreo por conveniencia.

\section{MARCO TEÓRICO}

Este trabajo incluye el aporte de investigaciones en relación con las variables de estudio, en García, R. C., Andino, M. R., y García, J. C. (2017) dentro del contexto ecuatoriano de la formación laboral de los docentes de educación y la PPP los resultados identifican que se han realizado cambios y reformas en la práctica, sin embargo aún persisten insuficiencias en la concepción, organización, orientación y desarrollo del proceso, así como la ausencia de estrategias y procedimientos que propicien una práctica reflexiva en correspondencia con las exigencias pedagógicas actuales.

De igual manera la investigación de Contreras (2014) cuya temática es una propuesta orientada al análisis de incidentes críticos auténticos en la formación de docentes de la Universidad Austral de Chile, los resultados expresan la concepción de una identidad del profesorado universitario como modelo de integración entre teoría y práctica pedagógica. El estudio de Huachara (2017) realizado en Lima- Perú a estudiantes de educación Inicial de una universidad privada, durante el periodo 2016 evidencia que existe una relación significativa entre la entre la creatividad y el desempeño en la PPP, el grado de correlación mediante el estadístico Rho de Spearman es ( $\mathrm{t}=0,453$; $\mathrm{p}<$ $0,05)$ lo que constituye que la creatividad es un elemento importante en el desarrollo de la PPP.

\subsection{Fundamentos teóricos de la educación inicial para la práctica pre profesional (PPP)}

El estudio evidencia la necesidad de fortalecer a nivel metodológico y tecnológico la propuesta didáctica de la PPP y los procesos de enseñanza-aprendizaje, se enfoca dentro de los cuatro pilares de la educación de Delors (1994) el aprender a conocer, aprender hacer, aprender a vivir juntos y el aprender a ser, documento de la UNESCO (1994).

\subsubsection{Modelo educativo Constructivista} El constructivismo es un modelo pedagógico que permite la generación del aprendizaje desde la experiencia mediante el "aprender haciendo", el estudiante es el centro del aprendizaje porque es quien construye sus conocimientos y el docente es el facilitador dentro del proceso de enseñanzaaprendizaje. De acuerdo con Torre y Vidal (2017) ser constructivista es estar a cargo de la construcción de su propia perspectiva del mundo que lo rodea a través de sus propias experiencias y esquemas mentales desarrollados, el modelo constructivista cuenta con tres principios básicos: aprender 
reconstruyendo los conocimientos, los contenidos y los procesos son complementarios y enseñar es favorecer el aprendizaje planificado y organizado.

Para Flores (2016) la teoría que más se aproxima al desarrollo adecuado de la práctica es la teoría constructivista especialmente enfocada hacia la generación del conocimiento. Pimienta (2012), (citado por Loor y Chávez 2020) fundamenta al constructivismo con un enfoque en el que las construcciones del conocimiento implican un rol directo del ser humano, este proceso inicia con lo adquirido en su "estructura cognitiva" producto de la experiencia durante su vida y el proceso interactivo con el otro, siendo este un encuentro producto del proceso educativo.

\subsubsection{Teoría del Aprendizaje Significativo}

El aprendizaje significativo de Ausubel (1963) quién sería el pionero de una teoría cognitiva (citado en Rodríguez, 2004) posibilita otorgar sentido a los conceptos y para conceptos, para dar cuenta de ellos el sujeto precisa conceptos y conocimientos previos, "esos conocimientos previos resultarán más elaborados en función de las situaciones en las cuales son usados, en esto reside la interacción que caracteriza el aprendizaje significativo en una perspectiva de progresividad y complejidad" (Moreira, 2017, p.8).

Dentro de la formación es preciso que el estudiante alcance niveles de creatividad importantes para cubrir las necesidades que surgen de la PPP "Para lograr ciudadanos creativos como se requiere en el presente siglo, es importante que, en las prácticas educativas, tengamos en consideración que potenciar la creatividad ofrece grandes posibilidades" (Chroback, 2017, p. 6).

\subsubsection{Teoría del Pensamiento Complejo} Brage y Cañellas (2017) en su estudio sobre el análisis al pensamiento complejo de Morin (1983) visibilizan el concepto de la praxis desde la acción, en Armijos, Hernández, y Sánchez (2017) sobre ésta temática refieren al pensamiento complejo como un paradigma epistemológico, mediante la reforma del pensamiento se conoce a la realidad como un sistema flexible en cambio continúo, siendo la toda la suma de las partes.

Este trabajo asume al modelo constructivista, la teoría del aprendizaje significativo de Ausubel (1983) e incluye la teoría del pensamiento complejo de Morin (1983). Las aplicaciones conjuntas de estas teorías permiten ese desarrollo eficaz de la didáctica y de un proceso de aprendizaje metacognitivo. "La complejidad no es una receta para conocer lo inesperado. Pero nos vuelve prudentes, atentos, no nos deja dormirnos en la mecánica aparente y la trivialidad aparente de los determinismos" (Morín,1976, p.75).

\subsection{Definición de práctica pre profesional (PPP)}

Entre las definiciones sobre la práctica PPP concebida como un espacio de aprendizaje para la formación futura de nuestros estudiantes, se ajustan a la idea central de éste trabajo las siguientes. La PPP es un momento de aprendizaje esencial en el proceso de formación profesional del estudiante universitario.

Para Davini (2015) la práctica es una realidad visible, representa el "hacer"; considera ser una visión restringida ya que no se puede hacer sin pensar. La práctica docente permite la formación de capacidades desde la experiencia, a partir de la construcción metodológica, la toma de decisiones en la acción, manejo y gestión de espacios, grupos de estudiantes, los recursos de enseñanza e información y los sistemas de evaluación. Las prácticas son resultados de los sujetos que involucran siempre el pensamiento y la valoración, así como las diversas nociones e imágenes sobre el mundo.

Loor, M. D. C., Loor, Y. C., Loor, M. M., y Carlos, C. C. (2020) hacen referencia a un concepto de la práctica reflexiva de Schön (1992) y Galindo (2012) desde un modelo que parta de la propia experiencia del estudiante más que de un proceso teórico, se refieren al "practicum reflexivo" donde el desarrollo de la práctica fluye por la acción. Sepúlveda (2016) considera que la PPP deberá integrar paulatinamente al estudiante dentro de la dinámica de la práctica, se deberá permitir el acceso del estudiante acorde al nivel de profundidad del conocimiento y ser capaz de 
dar una respuesta desde la experiencia a los problemas detectados en la misma.

La práctica plasma en una realidad tangible las experiencias personales, sociales, emocionales y culturales, su dinámica deberá ser flexible y adaptable a nivel curricular, temporal y espacial; es recrear el escenario de la vida profesional más próxima a la realidad desde una posición filosófica, epistemológica, metodológica, con valores y principios. Por lo tanto, este estudio aplicará el concepto de la PPP como una "acción" producto de un aprendizaje significativo y constructivo que permita el desarrollo teórico-práctico.

\subsection{Recursos didácticos digitales que favorecen la PPP}

Para Góngora y Martínez (2012) el diseño de aprendizaje basado en tecnologías (Computer Based Learning Design) favorece y potencia el trabajo de equipo entre los docentes que realizan la elaboración de los materiales, la diversidad de herramientas tecnológicas que existen actualmente para el diseño del aprendizaje optimizan la capacidad creadora de los docentes. Entre las diferentes variables que puedan influir en los procesos de diseño, planificación, ejecución y evaluación de la PPP encontramos las siguientes.

\subsubsection{El portafolio digital}

De acuerdo con Rodrigues (2013) el autor de un portafolio tiene el propósito de coleccionar sus trabajos y poderlos exponer a los demás mediante un registro detallado con la finalidad de indicar las competencias, destrezas y habilidades del autor. Siendo el portafolio educativo como un instrumento que facilita la selección de los productos educativos, con énfasis en la reflexión de la práctica educativa.

El portafolio como herramienta de aprendizaje presenta una evolución acorde a los cambios tecnológicos, por lo que deben corresponder a las necesidades de la sociedad del conocimiento, los portafolios en un inicio fueron únicamente físicos hoy son herramientas (portafolios digitales o eportafolios). Lamas y Vargas (2016) el portafolio tiene a más de fines formativos en cuanto a destrezas y fines evaluativos, es una herramienta que fomenta la reflexión potenciando las habilidades metacognitivas, tiene más fuerza cuando se cuenta con la participación del tutor y los compañeros.

Para Prendes y Sánchez (2008) surge de la necesidad de dar herramientas cualitativas o metodologías al docente y estudiantes para el proceso de evaluación y la verificación del avance de los conocimientos, logros, habilidades, destrezas y competencias; no puede ser una simple recolección de datos implica un proceso cronológico de reflexión acompañado de una autoevaluación, los autores refieren sobre el portafolio multimedia "webfolio" como un repositorio digital multimedia, permite inclusive el trabajo en pares académicos. Según Abrami y Barrett (2005) los e-portafolios tienen una gran ventaja en relación con el portafolio físico, ya que integran fácilmente en los aprendizajes dentro de los archivos multimedia, se puede verificar mejor el nivel de desarrollo de las competencias de los estudiantes y permite un proceso de retroalimentación permanente.En este estudio el portafolio digital es un soporte a todos los procesos educativos dentro del diseño, desarrollo, ejecución y evaluación de la PPP. El e-portafolio tiene un alcance con mayor cobertura y pueden estar estructurados en varios formatos, a nivel educativo interviene en el proceso de enseñanza-aprendizaje como el espacio que contiene las evidencias con calidad dentro del proceso educativo. En la investigación de Muñoz, Serván y Gómez (2019) de la Facultad de Ciencias de la Educación de la Universidad de Málaga-España, durante el curso académico 2016 de Educación Infantil se concluye que la aplicación del portafolio digital dentro del marco de una metodología constructivista, es una herramienta emergente a las necesidades educativas de este siglo.

\subsubsection{El juego-trabajo como medio interactivo}

El juego-trabajo como medio interactivo dentro del aprendizaje, facilita el desarrollo de actividades lúdicas y de conocimiento generando una práctica sobresaliente. Palacios (2019) define al juego como un problema donde interviene una decisión y existe más de un agente que lo define, éstas decisiones inciden sobre los otros jugadores, los juegos son educativos cuando los 
intereses de los agentes están contrapuestos. Para Monsalve (2013) la teoría de juegos interactivos estudia el comportamiento clave de los individuos en su interacción y sus decisiones impactarán sobre lo que el otro realice, por lo que el juego se desarrolla a medida de las interacciones de los agentes.

Un grupo interactivo dentro del trabajo de Sepúlveda (2016) es el que permite generar un nivel de responsabilidad importante en sus miembros, siendo los protagonistas de su aprendizaje y una parte fundamental del trabajo de sus compañeros y compañeras. La dinámica presente en el grupo interactivo se sustenta en el diálogo igualitario, el trabajo colaborativo, el debate $\mathrm{y}$ actitudes en desarrollo de principios y valores. Para Mosquera (2016) La metodología juego-trabajo permite activar el cumplimiento del ámbito que estimula el currículo de educación inicial siendo ésta actividad recreativa esencial para activar criterios dinámicos y creativos, porque el juego propicia la participación activa del estudiante y el docente diseña las estrategias considerando la actividad espontánea y libre del juego-trabajo.

Se lo debe incluir dentro de las actividades de planificación en todas las áreas del conocimiento, es un medio para generar los aprendizajes y la construcción de los mismos desde la acción lúdica que permite la organización, el respeto de turnos, el desarrollo metacognitivo, la asociación de ideas, la resolución de problemas, socializar, estimular la participación y es un espacio para la creatividad. En la investigación propuesta por la Universidad de Israel en Quito-Ecuador por Samaniego y Cabrera (2019) se aplicó un juego interactivo para reforzamiento del aprendizaje en niños de educación inicial, esta herramienta comprueba que el juego-trabajo facilita mediante un software mejorar la capacidad de atención y concentración de los niños de manera amigable y genera la interacción del niño con el aprendizaje lo que le permite un nivel superior de asimilación.

\subsubsection{La animación a la lectura mediante E-books}

El decálogo de animación a la lectura, en el décimo punto resalta la importancia de "Animar a leer no supone una competición con otros grupos, con otros centros educativos, con otras bibliotecas. No se trata de leer más que el otro grupo, el otro centro, la otra biblioteca, el otro barrio, el otro pueblo, la otra comunidad autónoma. Se trata de enseñar a disfrutar de la lectura, porque consideramos que es buena en sí" (Gómez, 2019, p.22).

Para animar a la lectura dentro de una propuesta de recursos educativos digitales, puede incluir una herramienta digital conocida como los E-books, que para Echeverri (2016) son la versión digital o multimedia de un texto o libro físico, lo que permite que puedan ser revisados mediante cualquier dispositivo digital.

Según Martínez (2012) la animación a la lectura es una serie de acciones que determinan la participación del grupo en relación a la lectura. La animación a la lectura necesita de estrategias que permitan generar el vínculo entre el material de lectura y el estudiante. Generalmente se apunta la animación a la lectura al acercamiento del niño o niña con los textos físicos y con las bibliotecas con la finalidad de ir despertando emociones y un determinado gusto por el aprendizaje desde el texto desde el componente más relevante que sería dentro del contexto lúdico como estrategia de animación.

Labra (2005) en su estudio indica que las actividades dirigidas a la animación de la lectura inician con los primeros "rudimentos lecto-escritores", así como también destaca la importancia de los cuentos. Se debe fortalecer la animación a la lectura especialmente en estos momentos en los que las TIC pueden ser antagónicos a la lectura y para ello este proyecto propone generar la animación a la lectura desde los E-books o libros digitales para acortar la brecha digital y motivar a la lectura como fuente del conocimiento, especialmente a las nuevas generaciones.

En esta investigación la animación a la lectura se centra como esa estrategia para acercar al aprendizaje inmerso en los textos ya sean físicos o digitales, en esta era en la que la tecnología lidera el tiempo y espacio de los niños, por ello desde ese contexto digital se puede alcanzar estrategias de animación a la lectura con el soporte de los E-books aproximándose el practicante a los intereses de los niños y niñas mediante las TIC. 
El estudio de Alcalá y Rasero (2004) en la Universidad de Extremadura de España, realiza una investigación sobre la fusión entre los libros y la tecnología de la información y comunicación, el estudio aplica un ejercicio sobre la lectura en España analizando el nivel de lectura en España desde los años 60's, durante las décadas de los 80's y 90's no hay un mayor crecimiento de la lectura, pero hacia el año 2 mil es visible la recuperación en la lectura de libros, esto debido a la intervención de la tecnología y animación a la lectura.

\subsubsection{Los bits de inteligencia en Web- albums}

Para Navarrete y Yagual (2017) los bits de inteligencia son considerados como un método didáctico que permite el desarrollo de la retención y de la memoria del niño desde la asimilación y acción de un objeto, son estrategias para los niños y niñas y consisten en la exposición de imágenes con la finalidad de mejorar la capacidad retentiva de conceptos, la asociación de palabras lo que permite prominentemente mejorar la destreza en su expresión oral, discriminación visual y su socialización.

En el estudio se definió que los bits de inteligencia pueden ir acompañado de los Web-álbum que para Echeverri (2016) son una herramienta que brinda un servicio donde se facilita subir y compartir información como fotos a través de un sitio web. En Céspedes (2019) son una herramienta precisa, apropiada y clara con un mensaje directo, la temática abordada puede estar dentro de los contenidos planificados para la sesión de trabajo de la maestra de inicial o bien dentro de un proyecto educativo, para activar los bits es preciso evitar ambiguiedades, fueron creados en la época pre digital que en la actualidad son completamente interactivos para los niños y niñas nativos digitales.

Los bits son láminas, dibujos, imágenes informativas que deben ser puntuales que denoten un significado a simple vista, con ésta herramienta digital los docentes pueden hacer uso de las láminas digitalizadas para trabajar y desarrollar destrezas metacognitivas, potencializar nociones, trabajar el lenguaje, la animación a la pre-lectura y pre-escritura. Permiten el desarrollo de la inteligencia y capacidades de los niños y niñas especialmente en los primeros años de formación inicial comprendidos entre los 3 a 5 años. El diseño, desarrollo, ejecución, evaluación y verificación de resultados de los bits de inteligencia mediante web-albums debe estar integrado a una planificación minuciosa donde se tengan objetivos claros.

Pomaleque (2019) desde su estudio en la Universidad del Altiplano, Puno-Perú sobre la influencia de los bits de inteligencia para el desarrollo de conocimientos de niños y niñas de cuatro años, concluye que la aplicación de los bits es positiva ya que permiten el desarrollo de conocimientos, éstas láminas o (bits) que presentan la información al niño o niña sobre una temática específica y de manera secuencial, cuya finalidad es lograr la retención de la imagen y en algunos casos del sonido, los bits permiten desarrollar habilidades y destrezas cognitivas.

\section{MÉTODO}

El estudio tiene un enfoque cuantitativo, de alcance descriptivo, correlacional y explicativo, con un diseño no experimental y transeccional (Hernández, Fernández y Baptista, 2014). La información recabada proviene de fuentes primarias no representativas, mediante la aplicación de una encuesta dirigida a estudiantes y a docentes tutores. Para medir cada una de las relaciones de las variables independientes con la dependiente, se aplicó el muestreo no probabilístico por conveniencia o estratégico en combinación con un muestreo por cuotas, con una muestra finita de 19 estudiantes y 2 docentes tutores.

Se ha decidido evaluar el grado de relevancia de los ítems con la aplicación de un instrumento de medición dentro de una prueba de validez de contenido bajo un análisis de tres expertos especialistas en didáctica de educación inicial.

Las hipótesis operativas fueron: el portafolio digital, el juego-trabajo como medio interactivo, la animación a la lectura mediante E-books y los bits de inteligencia en Web-albums, son recursos digitales que permiten mejorar la PPP de los estudiantes de la licenciatura de educación inicial de la Universidad Católica de Cuenca. El Modelo esquemático de la hipótesis es $\mathrm{Y}=\mathrm{f}(\mathrm{X} 1, \mathrm{X} 2$, 
$\mathrm{X} 3 \ldots . . . \mathrm{Xn}$ ), concluyendo con el modelo gráfico de la hipótesis.

En la investigación se utilizaron los siguientes métodos estadísticos: se aplicó el método de análisis descriptivo, con un análisis sociodemográfico del perfil del encuestado incluyendo las siguientes características (género, edad, escolaridad y estado civil). Haciendo uso de pruebas paramétricas para obtener los estadísticos descriptivos básicos de las variables (porcentajes, media, mínimo, máximo y desviación estándar), a una muestra de 21 personas. Para medir la confiabilidad del instrumento se aplicó el alfa de Cronbach la cual encontró valores dentro de los rangos aceptados igual a 0,70 .

\section{RESULTADOS}

El perfil del encuestado presenta los siguientes datos para las variables de control: el $95 \%$ del total de la población encuestada es de género femenino y el 5\% masculino, el $81 \%$ de los encuestados tienen una edad entre los 19 y 30 años, el $71 \%$ de la muestra responde estar cursando la formación de pregrado y el $90 \%$ de la población encuestada dice ser de estado civil soltero. Se aplicó el análisis de correlación bivariada de Pearson, dando como resultado un coeficiente $(\mathrm{R})$ de 0.90 lo que representa una correlación fuerte entre las variables.

Se aplicó el método de análisis factorial de componentes principales, el cual permitió conocer a priori el número de factores y establecer limitaciones sobre los elementos de la matriz, su aplicación permitió distribuir las dieciséis preguntas o reactivos en cuatro grupos, el resultado de la varianza total explicada arroja una probabilidad del $83,68 \%$ que es estadísticamente significativo. Dentro del componente rotado se puede evidenciar una carga factorial superior o igual a 0.6 se reflejan seis componentes, $\mathrm{C} 1$ contiene las preguntas con una carga factorial importante: P5(0,942), P9(0,970), P10 $(0,873)$ y $\mathrm{P} 11(0,863)$.

Tabla 1. Estimaciones de frecuencia por variables independientes.

\begin{tabular}{llcc}
\hline Variable & Escala de liker(1 al 5) & Frecuencia & Porcentaje \\
\hline $\begin{array}{l}\text { Portafolio } \\
\text { digital }\end{array}$ & $\begin{array}{l}\text { DA (De acuerdo) } \\
\text { TDA(Totalmente de } \\
\text { Acuerdo) }\end{array}$ & 8 & 38,1 \\
Juego-Trabajo & $\begin{array}{l}\text { DA (De acuerdo) } \\
\text { TDA(Totalmente de } \\
\text { Acuerdo) }\end{array}$ & 9 & 38,1 \\
Animación a la & Lectura & 9 & 42,9 \\
& $\begin{array}{l}\text { DA (De acuerdo) } \\
\text { Acuerdo) }\end{array}$ & 11 & 42,9 \\
$\begin{array}{l}\text { Bits de } \\
\text { inteligencia }\end{array}$ & $\begin{array}{l}\text { DA (De acuerdo) } \\
\text { TDA(Totalmente de }\end{array}$ & 6 & 52,4 \\
& Acuerdo) & 11 & 28,6 \\
\hline
\end{tabular}

Fuente: Elaboración propia, sistema SPSS.

En el análisis de frecuencia para cada una de las variables independientes, se extrajeron las variables más representativas dentro de la escala de Liker del 1 al 5, siendo 
1(Totalmente en desacuerdo), 2(En desacuerdo), 3(Neutro e indiferente), 4(De acuerdo) y 5(Totalmente de acuerdo). En referencia al porcentaje acumulado de la (Tabla 1) las variables presentan el mayor porcentaje en la escala de Liker con el nivel 4 (De acuerdo), el portafolio digital con un
$38,1 \%$, el juego-trabajo con 42,9\%, animación a la lectura con $52,4 \%$ y los bits de inteligencia con un 52,4\%. Lo que significa que las variables presentan una frecuencia alta en el nivel 4 (De acuerdo) de la escala.

Tabla 2. Estadísticos descriptivos

\begin{tabular}{llllll}
\hline & N & Mínimo & Máximo & Media & Desv. Desviación \\
\hline Portafolio digital & 21 & 1 & 5 & 3,81 & 1,401 \\
Portafolio digital & 21 & 1 & 5 & 4,14 & 1,062 \\
Portafolio digital & 21 & 1 & 5 & 4,24 & 0,889 \\
Portafolio digital & 21 & 1 & 5 & 4,14 & 0,964 \\
Juego trabajo & 21 & 1 & 5 & 4,00 & 1,342 \\
Juego trabajo & 21 & 1 & 5 & 4,14 & 1,014 \\
Juego trabajo & 21 & 1 & 5 & 4,29 & 0,956 \\
Juego trabajo & 21 & 1 & 5 & 4,43 & 0,926 \\
Animación a la & 21 & 1 & 5 & 3,95 & 1,024 \\
lectura & 21 & 1 & 5 & 4,22 & 0,835 \\
Juego trabajo & 21 & 1 & 5 & 4,24 & 0,762 \\
Juego trabajo & 21 & 1 & 5 & 4,26 & 0,690 \\
Juego trabajo & 21 & 1 & 5 & 4,28 & 0,618 \\
Juego trabajo & 21 & 1 & 5 & 4,30 & 0,545 \\
Animación a la & 21 & 1 & 5 & 4,31 & 0,473 \\
lectura trabajo & 21 & 1 & 5 & 4,33 & 0,400 \\
Juego trabajo & 21 & 1 & 5 & 4,35 & 0,328 \\
Juego trabajo & 21 & & & & \\
N válido (por lista) & & & &
\end{tabular}

Fuente: Elaboración propia, sistema SPSS-IBM

Según los resultados estadísticos descriptivos, la mayoría de los encuestados están de acuerdo con la hipótesis de estudio esto refleja la media y la desviación estándar de los reactivos (Tabla 2).

Tabla 3. Resultados en porcentaje por constructo y reactivo

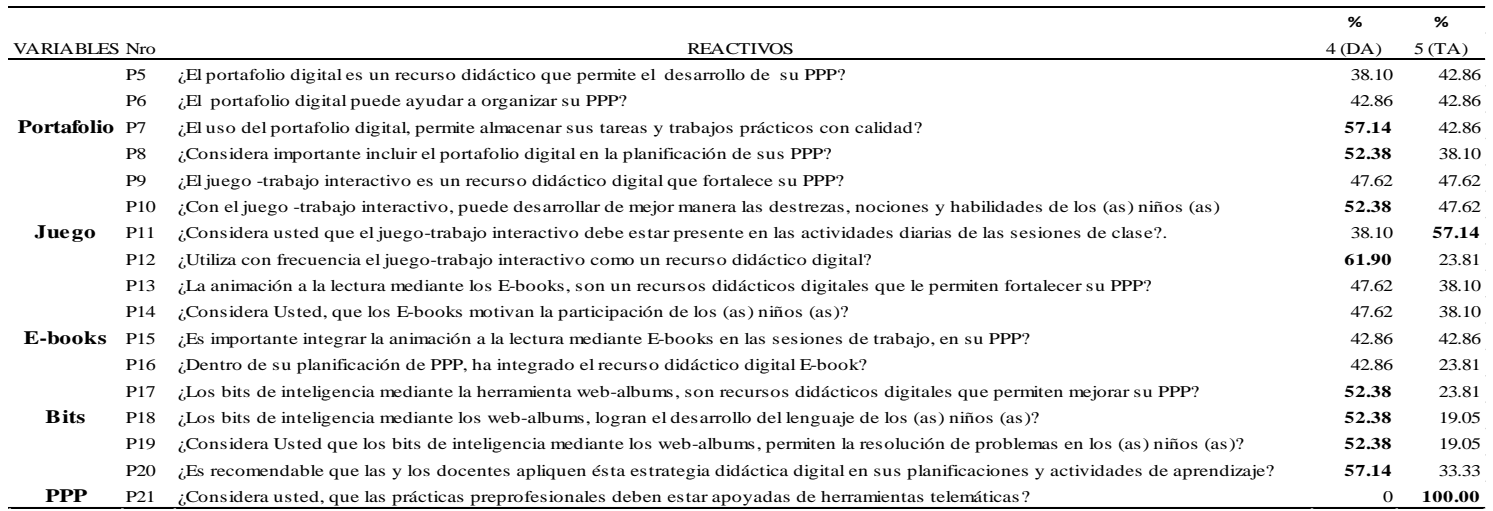

Fuente: Elaboración propia, encuesta.com.

Las variables independientes del estudio fueron diecisiete, divididas en cinco grupos denominados constructos; estos constructos del uno al cuatro contenían cuatro reactivos y el quinto con un reactivo relacionado a la variable (Y). Los reactivos tabulados en la (Tabla 3) van del 5 al 21; ya que los reactivos del 1 al 4 corresponden a las variables de control. Se realiza el análisis acorde a la escala de Liker del 1 al 5 tomando en cuenta 
los porcentajes más altos correspondientes a los niveles 4 (De acuerdo) y 5 (Totalmente de acuerdo), esto corrobora la información presente en la (Tabla 2).

Tabla 4. Confirmación de hipótesis

\begin{tabular}{lccc}
\hline Variables & $\mathrm{t}$ & \multicolumn{2}{c}{ Sig. } \\
\hline Portafolio digital & 4.336 & .001 & .555 \\
Juego trabajo & 6.147 & .000 & .491 \\
Animación a la lectura & 5.576 & .000 & .344 \\
Bbits de inteligencia & 3.755 & .002 & .360 \\
\hline
\end{tabular}

Fuente: Elaboración propia, sistema SPSS-IBM

\section{Discusión}

Para confirmar la hipótesis del presente estudio, se aplicó la técnica econométrica de regresión lineal simple en el sistema (SPSS de IBM) véase (Tabla 4), en la técnica (t de student) cuyo resultado debe ser mayor o igual a 1,96, las variables explicativas impactan significativamente con un 4,336 el portafolio digital, en un 6,147 el juego trabajo, la animación a la lectura en un 5,576 y los bits de inteligencia en un 3,755 en

El nivel de confiabilidad del ( $R$ cuadrado) está dentro del rango 0 a 1 siendo el de mayor significancia el portafolio digital.

El resultado de la variable dependiente, demuestra el cumplimiento total de la hipótesis, los datos de la investigación de Lima, Perú de Huachara (2017) refieren a la relación significativa de la creatividad y el desempeño de la PPP, acorde a los resultados de la investigación aplicada en las 21 encuestas, véase la (Tabla 3 ) en el reactivo $\mathrm{P} 21$ el nivel responde en su totalidad al 5 Totalmente de acuerdo (TA) en un $100 \%$, ya que la PPP debe estar apoyada en herramientas telemáticas las cuales desarrollan un mejor diseño, creatividad y ejecución en el proceso. En cuanto a los resultados sobre las variables independientes cabe señalar que en su gran mayoría los reactivos se encuentran en el nivel 4 De acuerdo (DA) y 5 Totalmente de acuerdo (TA), véase (Tabla 4) lo que permite comprobar la hipótesis de éste estudio en el que se consideran a los recursos didácticos digitales para mejorar la PPP en el contexto de la emergencia sanitaria COVID-19.

Si se toma la primera variable con relación a los resultados de Muñoz, Serván y relación con la variable dependiente PPP. La significancia de las variables independientes es menor a 0.05 siendo estadísticamente significativas. El coeficiente de relación entre las variables es de $\mathrm{R}=0.55$ para el portafolio digital, $\mathrm{R}=0.49$ para el juego trabajo, en $\mathrm{R}=0.34$ para la animación a la lectura $\mathrm{y}$ un $\mathrm{R}=0.36$ en los bits de inteligencia; especialmente el portafolio digital y el juego trabajo presentan una correlación fuerte entre las variables.

Soto (2019) el estudio confirma que la aplicación del portafolio digital como metodología constructivista está acorde a las demandas educativas del siglo XXI, corroborando con éste estudio la presente investigación en el reactivo P8 en el nivel 4 (DA) es importante incluir el portafolio digital en la planificación de la PPP en un $52,38 \%$. En cuanto a la segunda variable los resultados del estudio de Samaniego et al. (2019) el juego interactivo es un software que mejora la capacidad de atención en la educación inicial, con esto se confirma el reactivo $\mathrm{P} 12$ en el nivel 4(DA) en un $61,90 \%$ donde se explicita que el juego trabajo interactivo es utilizado como un recurso digital eficaz en la PPP.

La investigación de Abramami y Barrett (2005) confirma que hacia los años 2 mil el nivel de lectura en España aumenta considerablemente debido al avance de la lectura digital y la animación a la lectura, este estudio a la vez confirma la variable de estudio en el reactivo $\mathrm{P} 13$ donde los recursos didácticos digitales mediante la animación a la lectura y los E-books fortalecen la PPP en un $47,62 \%$ en el nivel 4 (DA) y en un $38,10 \%$ con respuestas en el nivel 5 (TDA). En referencia a la cuarta variable acorde con 
los resultados del estudio de Pomaleque (2019) sostiene que los bits de inteligencia desarrollan significativamente las habilidades y destrezas cognitivas de los niños y niñas de 4 años, este estudio en su reactivo P20 recomienda en el nivel 4 (DA) que las y los docentes apliquen los bits de inteligencia en sus planificaciones $\mathrm{y}$ actividades de aprendizaje en un 57,14\% y en un $33,33 \%$ en el nivel 5 (TDA), con esto se comprueba que ésta variable es muy significativa dentro de éste estudio.

\section{CONCLUSIONES}

Este proyecto de investigación ha permitido confirmar la necesidad de plantear estrategias didácticas como recursos

\section{Recomendaciones}

Luego de verificar que la hipótesis de estudio ha sido confirmada y que existen varios estudios que la confirman, se recomienda continuar con ésta línea de investigación para futuros estudios, analizando la importancia de otras estrategias y recursos didácticos digitales que contribuyan al fortalecimiento $y$ adecuado desarrollo del sistema educativo en general, se deberá indagar sobre el digitales dentro del desarrollo de la práctica pre profesional, considerando el escenario que plantea la emergencia sanitaria COVID19.

Se identificaron las variables dependientes de estudio y éstas confirman la hipótesis considerándolas como importantes en el diseño, planificación y ejecución dentro de las prácticas pre profesionales. La encuesta diseñada y aplicada a los 19 estudiantes y 2 docentes tutores verifican la importancia de integrar estas herramientas en el contexto de la pandemia, se constata que dentro de la escala de Liker el nivel 4 (De acuerdo) es un nivel representativo dentro de la formulación de la encuesta, ya que en su totalidad los reactivos tuvieron el mayor porcentaje en este nivel.

desarrollo, aplicación de portales y softwares educativos.

Los docentes de las licenciaturas de educación inicial deben considerar el incluir definitivamente éstas estrategias didácticas digitales especialmente en estos momentos en los que la tecnología alcanza un mayor nivel de cobertura en relación a priori a la emergencia sanitaria. 


\section{REFERENCIAS}

Abrami, P. y Barrett, H. (2005). Directions for Research and Development on Electronic Portfolios. Canadian Journal of Learning and Technology, 31(3), 1-12. En: https://www.learntechlib.org/p/43165/

Alcalá Caldera, J., \& Rasero Machacón, J. (2004). El papel de las TIC en la animación a la lectura. RELATEC, 3(1), 395-416. En: https://relatec.unex.es/article/view/141

Armijos, C. E. G., Hernández, M. W. H., \& Sánchez, R. E. R. (2017). Principios epistemológicos para el proceso de la enseñanza-aprendizaje, según el pensamiento complejo de Edgar Morin. Pueblo Continente, 27(2), 471-479.En: http://journal.upao.edu.pe/PuebloContinente/article/view/699

Bizberge, A., \& Segura, M. S. (2020). Los derechos digitales durante la pandemia COVID-19 en Argentina, Brasil y México. Revista de Comunicación, 19(2), 61-85. En: http://www.scielo.org.pe/scielo.php?pid=S1684-09332020000200061\&script=sci_arttext

Brage, L. B., \& Cañellas, A. J. C. (2017). Epistemologías de la complejidad y educación. (1 ${ }^{\mathrm{a}}$. ed.). Octaedro.

Consejo de Educación Superior del Ecuador (CES) (2020) Resolución RPC-SE-03-No.046-2020. Normativa transitoria para el desarrollo de actividades académicas en las Instituciones de Educación Superior, debido al estado de excepción decretado por la emergencia sanitaria ocasionada por la pandemia de COVID-19. En: https://www.epn.edu.ec/wpcontent/uploads/2020/04/normativa_transitoria_rpc-se-03no.046-2020.pdf

Contreras, C. (2014). El desarrollo docente del formador de profesores: una propuesta orientada hacia el análisis de incidentes críticos auténticos. Estudios pedagógicos (Valdivia), 40(n, especial), 49-69. En: https://scielo.conicyt.cl/scielo.php?script=sci_abstract\&pid=S071807052014000200004\&lng=es\&nrm=iso

Chrobak, R. (2017). El aprendizaje significativo para fomentar el pensamiento crítico. Archivos de Ciencias de la Educación, 11(12), 1-12. En: file:///C:/Users/UCACUE/Downloads/8210-Texto\%20de1\%20art\%C3\%ADculo-19596-110-20171206.pdf

Davini, M. (2015). La formación en la práctica docente. (1 $1^{\mathrm{a}}$. ed.). Paidós.

Delors, Jacques (1994). Los cuatro pilares de la educación, en La Educación encierra un tesoro. El Correo de la UNESCO. En: https://www.uv.mx/dgdaie/files/2012/11/CPP-DC-DelorsLos-cuatro-pilares.pdf

Echeverri, L. (2016). Recursos educativos digitales para preescolar y primaria. (pp. 25). UNIREMINGTON

García, R. C., Andino, M. R., \& García, J. C. (2017). Tendencias de la formación inicial docente en Ecuador con énfasis en la práctica preprofesional pedagógica. Killkana sociales: Revista de Investigación Científica, 1(3), 61-74. En: https://killkana.ucacue.edu.ec/index.php/killkana_social/article/view/64

Gómez, A. (2019). Platero: revista de literatura infantil-juvenil y animación a la lectura, 222(10) 22-28. En: https://dialnet.unirioja.es/ejemplar/531587

Góngora Parra, Y., \& Martínez Leyet, O. L. (2012). Del diseño instruccional al diseño de aprendizaje con aplicación de las tecnologías. Teoría de la Educación, educación y cultura en la sociedad de la información.13(3), 342-360. En: https://gredos.usal.es/handle/10366/121837

Huachara Martínez, E. (2017). La creatividad y el desempeño en la práctica pre profesional en estudiantes de IX y X ciclo de Educación Inicial en una universidad privada. Lima. (Tesis inédita). Magister en docencia universitaria. Escuela de Posgrado. Universidad Cesar Vallejo, Lima, Perú. En: https://repositorio.ucv.edu.pe/handle/20.500.12692/7739?show=full

Hernandez, R. Fernández, C., y Baptista, P. (2014). Metodología de la investigación. (6a. ed.). McGraw-Hill.

Labra, J. P. (2005). Animación a la lectura y TIC: creando situaciones y espacios. Sociedad lectora y educación, nro. extraordinario 1, 255-279. En: https://dialnet.unirioja.es/servlet/articulo?codigo $=1332482$ 
Labra Godoy, L. P., \& Fuentealba Jara, R. (2011). Construcción de conocimiento profesional docente: el caso de la formación en la práctica. (Tesis inédita). Doctorado en educación. Universidad Academia de Humanismo Cristiano, Santiago, Chile. En: http://bibliotecadigital.academia.cl/xmlui/handle/123456789/679

Lamas, P., \& Vargas-D'Uniam, J. (2016). Los niveles de reflexión en los portafolios de la Práctica Pre Profesional Docente. REDU. Revista de Docencia Universitaria, 14(2), 57-78. En: https://polipapers.upv.es/index.php/REDU/article/view/5680

Loor, M. D. C., Loor, Y. C., Loor, M. M., \& Carlos, C. C. (2020). Fundamentos teóricos del constructivismo y el enfoque reflexivo y su aporte en el perfeccionamiento del proceso de las prácticas pre profesionales. Revista de Investigaciones en Energía, Medio Ambiente y Tecnología, 5(1), 10-14. En: https://revistas.utm.edu.ec/index.php/Riemat/article/view/2497

Martínez, L. J. (2012). La animación a la lectura en las bibliotecas La construcción de un camino hacia la lectura. Boletín de la asociación andaluza de bibliotecarios, 27(103), 59-78. En: file:///C:/Users/UCACUE/Downloads/Dialnet-

Mena Céspedes, G. M. (2019). Bits de inteligencia digital en el desarrollo de la competencia de los niños del nivel inicial. (Tesis inédita). Especialidad en Educación Inicial. Universidad Nacional de Tumbes, Piura, Perú. http://repositorio.untumbes.edu.pe/handle/UNITUMBES/1330

Monsalve, S. (2003). John Nash y la teoría de juegos. Lecturas Matemáticas. Revista Universitaria de la Universidad Nacional de Colombia-Bogotá, 24, (1), 137-149. En: http://www.cienciared.com.ar/ra/usr/4/26/john nash y la teor a de juegos.pdf

Moreira, M. A. (2017). Aprendizaje significativo como un referente para la organización de la enseñanza. Archivos de Ciencias de la Educación, 11(12), 1-16. En: file:///C:/Users/UCACUE/Downloads/8208-Texto\%20del\%20art\%C3\%ADculo-19561-210-20171206.pdf

Morin, E. (1976). Introducción al pensamiento complejo. En: http://cursoenlineasincostoedgarmorin.org/images/descargables/Morin_Introduccion_al_p ensamiento_complejo.pdf

Mosquera Moreno, R. T. (2016). Inducción al currículo de educación inicial y su metodología juego trabajo en las instituciones educativas del cantón Machala. (Tesis inédita). Licenciatura en Ciencias de la Educación, Mención Educación Inicial. Universidad Técnica deMachala, Machala, Ecuador. En: http://repositorio.utmachala.edu.ec/handle/48000/9171

Muñoz González, L. D. L. C., Serván Núñez, M., \& Soto Gómez, E. (2019). Las competencias docentes y el portafolio digital: Crear espacios de aprendizaje y evaluación en la formación Inicial del profesorado. Un estudio de casos. Revista Iberoamericana de Evaluación Educativa, 12(2), 111-131. En: https://revistas.uam.es/riee/article/view/riee2019.12.2.006

Navarrete García, T. Z., \& Yagual Yagual, J. N. (2017). Influencia de los bits de inteligencia en la calidad del desarrollo de aprendizaje en niños de 4-5 años. (Tesis inédita). Licenciatura en Ciencias, Mención Educación en Párvulos. Universidad de Guayaquil, Guayaquil, Ecuador. En: http://repositorio.ug.edu.ec/handle/redug/23168

Olmedo-Torre, N., \& Farrerons-Vidal, O. (2017). Modelos constructivistas de aprendizaje en programas de formación. OmniaScience. En: https://www.google.com/search?q=OlmedoTorre\%2C+N.\%2C+\%26+Farrerons-Vidal\%2C+O.+(2017)

Palacios, L.A. (2019). Teoría de juegos, planeación y estrategia. Ciencia y Datos. Ciencia \& Datos. Recuperado en: https://medium.com/datos-y-ciencia/teoria-de-juegosplaneaci\%C3\%B3n-y-estrategia-73fc530c7123

Pomaleque, M.C. (2019). Aplicación de los bits de inteligencia para el desarrollo de conocimientos sobre los animales de la sierra de Puno en los niños y niñas de 4 años de la Institución Educativa Inicial No 649 Ipacuña del Distrito de Tirapata - 2017. (Tesis inédita). Especialidad en Educación Inicial. Universidad del Altiplano, Puno, Perú. En: http://repositorio.unap.edu.pe/handle/UNAP/11412

Prendes Espinosa, M. P. y Sánchez Vera, M.M. (2008). Portafolio Electrónico: posibilidades para los docentes. Pixel-Bit. Revista de Medios y Educación, 32, 21-34. En: https://www.redalyc.org/pdf/368/36803202.pdf 
Rodrígues, R. (2013). El desarrollo de la práctica reflexiva sobre el quehacer docente, apoyada en el uso de un portafolio digital, en el marco de un programa de formación para académicos de la Universidad Centroamericana de Nicaragua. (Tesis inédita). Doctorado en Educación y Sociedad. Universidad de Barcelona, Barcelona, España. En: http://www.redfforma.cl/documentos sitio/43828 RRODRIGUES TESIS.pdf

Rodríguez, M. (2004). La teoría del aprendizaje significativo. Concept Maps: Theory, Methodology, Technology. Conference on Concept Mapping. Pamplona, España. En: http://cmc.ihmc.us/Papers/cmc2004-290.pdf

Samaniego, V. J. C., \& Cabrera, R. M. O. (2019). Juego interactivo para reforzamiento del aprendizaje en niños de educación inicial. Apropiación, generación y uso edificador del conocimiento de estudiantes sentipensantes ( $1^{\mathrm{a}} \mathrm{ed}$.). REDIPE.

Sepúlveda, M. D. P. (2016). La enseñanza de comprensión y producción de textos breves mediante la metodología de grupos interactivos. Revista de Estudios y Experiencias en Educación, 6(12), 131-141. En: http://www.rexe.cl/ojournal/index.php/rexe/article/view/188

Torres, R.M. (2020). Otra educación. En: https://otra-educacion.blogspot.com/2020/04/elecuador-y-la-digitalizacion-de-la.html.

Zapata, B. E., \& Ceballos, L. (2010). Opinión sobre el rol y perfil del educador para la primera infancia. Revista Latinoamericana de Ciencias Sociales, Niñez y Juventud, 8(2), 10691082. En: https://www.redalyc.org/pdf/773/77315155021.pdf 